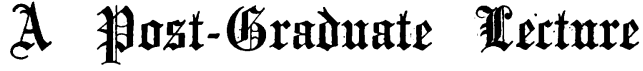

THE VARIETIES OF MALIGNANT
ENDOCARDITIS.

Delivered at the London School of Clinical Medicine (SEamen's Hospital).

BY FREDERICK TAYLOR, M.D., F.R.C.P.,

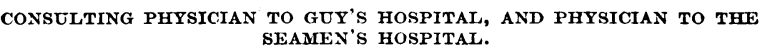

Gentlemen,-I propose to bring to your notice some cases of malignant endocarditis, with the object of showing you what a great variety of clinical symptoms such cases present, and of discussing the possibility of classifying them.

In malignant endocarditis we have a condition of septicaemia associated with microbic endocarditis, and attended, as a rule, to which exceptions are rare, by pyrexia. The microbic process taking place in the current of the circulation, and bringing about as it does the destruction of the valves, allows the distribution of the micro-organisms, and of fragments of fibrin and of necrosed valve tissue to all parts of the body; and such organisms or particles become impacted in vessels, and produce the phenomena of infarction, haemorrhage, suppuration, and interference of function of the parts whose vessels are obstructed.

Thus we have as clinical features of the disease: $(a)$ The evidence of endocarditis, in murmur or changes in the cardiac muscle; $(b)$ the evidence of sepsis-for example, pyrexia; $(c)$ the evidence of obstruction of vessels (embolism) by coarse particles.

If the evidence under these three heads is full and unmistakable, there is no difficulty in coming to a conclusion as to the nature of the illness; but we are obliged to admit that, while pyrexia in some form is the most constant sign, the evidence of a cardiac lesion and the evidence of coarse embolisms are frequently wanting. And, further, it must be recognized that even when an individual is known to have a cardiac lesion, the supervention of pyrexia does not at once show that this cardiac lesion has taken on septic or infective characters, because it is obvious that such a person is not protected from typhoid fever, nor from tuberculosis, nor from other infections causing pyrexia and death. Other important facts in its history are: (1) That its duration is extremely variable, ranging in different cases from a week or ten days to six, nine, or even twelve months; (2) that the infective phase may come on at any period in the course of valvulair lesion - either quite early, when the lesion is compensated and the patient is unaware that he has a lesion, or later, when the patient is bedridden, in the last stages of orthopnoea and dropsy; (3) that the chief clinical manifestations of the disease may be in different parts of the body, dependent upon the vessels into which the emboli are carried and upon the character and virulence of the organisms which accompany them. Thus, in one case a hemiplegia may occur, in another meningitis, in another ocular lesions, in another haematuria, in another a splenic abscess, in another cutaneous haemorrhages, in another gangrene of an extremity, in another pneumonia, and in others combinations of two or more of the preceding.

\section{Crassification of Cases.}

Hence it is obvious that the very great variety is observed in the cases, and that the more we know of the disease, the more difficult does it become to divide up the cases into really distinct groups-in other words, to classify them successfully.

The attempt has, of course, been made, and the classification hitherto adopted has been generally into four groups, namely, a typhoid, a pyaemic or septic, a cardiac, and a cerebral group. I have never regarded this as quite satisfactory; as a very little experience of cases shows one that a given case may fairly claim to be put in two, if not in three, groups at the same time.

I am unwilling to raise difficulties, and if the use of an even imperfect classification helps one's memory, or powers of diagnosis, I would be the last to deprecate it but a classification that does not cover the whole field may do harm by leading one to exclude a case which does not fall into one of the given groups, and a bad cross classification is practically useless, or misleading, when required for descriptive purposes. I may be permitted a few words on the grouping hitherto adopted.

The term "typhoid" is vague; it should mean "like typhus," a disease of which the English practitioner now sees little. If it means "like typhoid," it may ke used to mean this in regard to its distinctive features, such as spots, diarrhoea, and enlarged spleen, or in regard to those general advanced conditions of prostration and asthenia which one sees not only in typhoid but in other toxic conditions, such as fatal small-pox, scarlatina, pyaemia, or indeed septicaemia of any kind.

A pyaemic type can only differ from the worst form of the typhoid group in the occurrence of rigors, or in the presence of abscesses. When pyaemia was common it was often confounded with typhoid fever; the rigors of pyaemia are variable; and rigors occur even in otherwise typical cases of typhoid fever without obvious cause and without ill effect. They are very variable in malignant endocarditis, and they are often absent; they occur at rare intervals in some prolonged cases of the disease. I have known them (Case III) almost daily for one week or ten days of the illness, and then absent the succeeding three weeks, death following without their reappearance. They are not con. fined to cases with foci of suppuration even in malignant endocarditis ; and we know, of course, that they are characteristic of malaria without suppuration, and usher in a number of infective disorders in which suppuration holds no place except as a complication; and then rigors may be absent. The suppurative stage of small-pox, for instance, is not characterized by rigors, while the onset is. The practical value of insisting on a resemblance to pyaemia is at the present day small, when so few cases of pyaemia are seen. In the post-mortem reports of Guy's Hospital, in the years 1903, 1904, 1905, were found 24 cases of pyaemia; and I gather from my experience as an examiner in medicine for a qualifying degree that few students have a practical knowledge of the post-mortem conditions of this disease. To describe a pyaemic group is almost the same as to describe ignotum per ignotius.

"Septic" is a term of considerable ambiguity. Septicaemia has been defined as a disorder in which organisms circulate in the blood, and it is distinguished from the condition in which organisms are confined to a surface wound, and only toxins circulate in the blood. Malignant endocarditis itself is essentially a septic endocarditis, and is frequently called by that name. To attempt to distinguish a par ticular group of the cases as septic, implying therefore that the others are not septic, does not seem a very satis factory proceeding. With surgeons sepsis is the equivalent of suppuration. If a wound suppurates, it has become septic ; septic, that is, pyogenic, organisms have entered and pus is the result. But if all pyogenic organisms are septic, surely all septic organisms are not pyogenic ; and if sepsis means suppuration, certainly that is a process which is absent from many cases of malignant endocarditis.

Passing now to the cardiac type, the inadequacy of the term as a distinctive one is obvious; the heart is involved of course, in all cases of malignant endocarditis-its implication is an essential part of the disease, and the term is nonsense without it. I understand the term to mean a case in which the valvular lesion has caused mechanical effects and disabilities, and the patient is manifestly suffering from heart disease, want of compensation or hypertrophy and dilatation, before the septic condition is developed. But is the term, then, to be limited to bedridden cases with dropsy, or may it be extended to cases in which a lesion has caused hitherto only a little shortness of breath, palpitation, or cardiac pain? Where is the line to be drawn? Assume, then, that we can agree as to whether the group is to include few or many cases, some of these cases will certainly have the characteristics of the septic group, others the characteristics of the typhoid group; others, again, may have cerebral complications, and come under the head of the cerebral group. In other words, we have here a cross classification.

Lastly, the cerebral group may be said to include cases in which meningitis is the terminal feature of the case. But I imagine if a case were complicated with embolic 
hemiplegia, it might also be placed in the same group; and in either of these cases fully developed cardiac lesions may be present, and probably septic phenomena as well.

Hence one sees how difficult is the problem of classification. Classification is an artifice, almost a trick. Nature does not classify; she produces almost continuous series. Man classifies as a feeble arrangement, in order to bring within the scope of his intellect or the power of his memory the innumerable variety of facts that exist. In order to classify one must know, and the more one knows the more impossible it is to separate the numerous things from one another into distinct groups. This is increasingly obvious in any department of medicine or bacteriology. And it is true of malignant endocarditis.

\section{Symrtoms.}

There is almost infinite variety, at any rate very great variety, in the symptoms and in the duration of malignant endocarditis; would there were more variety in its outcome and termination!

One of the first things to recognize is that malignant endocarditis may give rise to a prolonged hectic, or if you like septic, fever or pyrexia lasting several months, with no cardiac symptoms, and no subjective troubles other than weakness, until perhaps towards the end, when some of the results of emboli may become pronounced. The following case is one in point:

$$
\text { CASE I. }
$$

A lady, aged 60, on the 5th day of the month found out her self that her temperature was above the normal. Her medical attendant, Dr. A. Scott, examined her and found that she had a attendant, Dr. A. Scott, examined her and found that she had a systolic apex murmur. The temperature continued to range to this discovery the lady had no idea that there was anything to this discovery the lady had no idea that there was anything wrong with her heart, except that for some time she had been conscious.

She was a healthy-looking, well preserved, cheerful woman; the heart's apex was half an inch outside the nipple, and a systolic murmur was audible at the apex, in the axilla, and on both sides behind. There were no rigors, the spleen could not be felt, and the only confirmation of the suggested diagnosis was the fact that we could not feel a pulse in the right dorsalis pedis artery. Four weeks later I saw her again. The temperature had continued almost uniformly between $98.5^{\circ}$ in the morning and $100^{\circ}$ or $101^{\circ}$ in the evening. There had been no rigorng, and 100 . " sweats; at most a "creepy feeling" when the temperature rose in the evening, and a little moisture of the skin. There were no rose spots; neither the spleen nor the liver could be felt; the pulse of the dorsalis pedis artery was absent; the murmur was unaltered. The blood had been searched for streptococci, and they had not been found.

With the exception of the first ten days, there was an almost uniform morning temperature of $98.5^{\circ}$ to $99^{\circ}$, and an evening temperature of $100^{\circ}$ to $101^{\circ}$, with few deviations from this rule. She died seven weeks later, the illness having a total duration of twenty-two weeks. There was almost no variation in the symptoms throughout; she became gradually weaker, and towards the end had an attack of dyspnoea and irregularity of the pulse, from which she ipartially recovered before the end came.

I note here especially the remarkable-I do not say extraordinary, because $I$ think it is common, but the remarkable-uniformity of the temperature for weeks and months. It has been said the temperature of malignant endocarditis is characterized by 'irregularity. In this group of cases I should say it was characterized by uniformity and regularity, each day precisely like the preceding and the following, and in that respect closely resembled prolonged cases of tuberculosis.

Now this is one familiar type of the disease. It lasted here five and a half months; it may last nine months or more-a uniform hectic temperature and a cardiac lesion which is well compensated, and which does not cause subjective or objective heart disease. The cases are not always so free from accompanying symptoms or the evidences of secondary lesions. Some patients are anaemic; in some the spleen is early felt; in others albuminuria appears; in others the absence of pulse from two or three of the superficial arteries may be demonstrated; in some rigors may occur, not constantly, but from time to time, singly at intervals of two or three weeks perhaps, and not referable to 'any external or internal causative circumstance.

Now if it is thought that this really resembles typhoid fever more than it does, say, the fever of tuberculosis, or broncho-pneumonia, it may be right to speak of it as a typhoid form; but the only form of typhoid it resembles is that in which spots are absent, diarrhoea does not exist, the spleen cannot be felt, and the patient's sensorium is clear and healthy - that is, it resembles the least typical form of typhoid, in which the diagnosis can only be made by Widal's test and not always by that. Repeated rigors, if they occur, may seem to justify the term " pyaemic," but if they are infrequent I should say not-and apart from the rigors there is little resemblance between these longlasting, chronic, comparatively mild conditions of infective endocarditis, and the rapidly fatal, adynamic toxaemic condition which acute pyaemia presents.

Probably it will be best to describe them as a simply pyrexial form. But there are infinite gradations between these long-lasting cases and those in which the duration is short, the fever intense, and the complicating conditions numerous.

The following illustrates a somewhat more rapid form:

\section{CASE II}

I saw this gentleman with Dr. W. Bramley Taylor. He was aged 39, and on November 1st he was taken ill with fever and headache. On the 3rd he had a rather sharp rigor, on the 5th and 6th each, and then milder but still a definite shiver; the bowels were rather confined, and laxatives were given. The temperature continued high for a few days, and then fell to normal. He was up on the 9 th and 10 th and on the 12 th he went out. That evening he was again ill, the temperature rose to $101.4^{\circ}$; the same on the 13th, and I saw him on the 14th.

Now here is the interesting part in the history. As a boy he had chorea occurring frequently; about twelve years ago he had rheumatic fever, and again six years later. After the chorea he was told that his heart was not sound. But he had up to the was told that his heart was not sound. But he had up to the
date of his illness not found it necessary to behave otherwise date of his illness not found it necessary

On examination, the heart's impulse was rather forcible there was a rough presystolic murmur at the apex, and a sof systolic and high-pitched diastolic murmur at the base. Dr. Bramley Taylor told me these murmurs had become louder during his illness. I found the spleen 1 즐 in. below the costal margin; there was no diarrhoea, no iliac gurgling, no rose spots, no optic neuritis, and the pulses were normal everywhere Four days later there were some pink spots, which I could not positively say were of typhoid origin, and there were some petechiae. His wife said he had a little difficulty with his speech on the 14th, but this was not confirmed by his doctor. The murmurs were not quite so pronounced.

On the 30th the murmurs were again well marked, the spleen was just perceptible, the liver was felt $2 \frac{1}{2}$ in. below the ribs; was just perceptible, the liver was felt $2 \frac{1}{2}$ in. below the ribs;
there was no pallor. The temperature, which had fallen to normal on the 3rd, had since been gradually rising like a low normal on the 3rd, had since been gradually rising like a low
course of typhoid. Widal's test was negative. He was seen course of typhoid. Widal's test was negative. He was seen
also by Dr. Mitchell Bruce, who confirmed the diagnosis. He also by Dr. Mitchell Bruce, who confirmed the diagnosis. He nounced modification of the temperature. He gradually got weaker without any great rise of temperature or any fresh symptoms, and died on March 4th, that is, after an illness of four calendar months' duration.

If we try to classify this case, the only justification for calling it typhoid is that it was pyrexial; the only justification for suggesting it was pyaemic is the fact that there were a few rigors at the commencement. The diagnosis was based on the existence of cardiac murmurs, on a temperature which did not run the true typhoid course, on the slight enlargement of the spleen, and on the few petechial spots which were present early in the case. If such a case is strongly suggestive of a negative typhoid in the first two weeks, it loses its resemblance as it proceeds into the fourth and later weeks.

Some years ago the following case occurred:

\section{CASE III.}

A woman, aged 50, was admitted under my care into Guy' Hospital on December 16th. She was first seen by a doctor on the 10th, when she had pain in the appendical region with some fever; the temperature, however, was normal on the 13th and 14th, and rose again suddenly on the night of the 15th. On 14th, and rose again suddenly on the night of the 15th. On and tenderness in the right flanks and iliac region, but the and tenderness in the right flanks and iliac region, but the abdomen was supple, and a careful examination of the righ sign whatever of trouble in that spot. The temperature continued high from the $16 \mathrm{th}$ to the $22 \mathrm{nd}$, and she had in that time five or six rigors; from the 23rd onward there were no more rigors, and the temperature was chiefly at the level of $101^{\circ}$ or $100^{\circ}$. There was no cardiac murmur; the pelvic organs were irregut râles were heard in the and spleen were slightly enlarged; a trace of albumen appeared in the urine, and she died on January 12th.

The post-mortem examination showed vegetations on thickened mitral valve, and much thickened aortic valves. Thickened mitral valve, and much thickened aortic valves. There were several small areas of red hepatization in the right appearance of infarcts, nor were they at all softened. The 
kidneys were large, with many old scars and recent petechia haemorrhages in small number. The spleen was large and soft and the liver showed a tight-lace constriction. The appendix vermiformis was perfectly healthy, and there was no peritonitis. There was a calculus in the right kidney.

This case may, perhaps, be regarded as of pyaemic type, but if so, solely on account of the rigors, which occurred in the first week of her stay in hospital, and which were absent entirely during the last three weeks of her life.

Much more closely resembling a case of typhoid fever is the following case:

CASE IV.

A man, from 45 to 50 years old, big, stout, artisan br occupation, and apparently a free liver, was seen by me with Dr. Rose of Norwood. He had been febrile eight days, and was now half drowsy and delirious, with a pulse of 120. "The heart sounds were healthy; there were râles at both bases; the liver was hard and large; the condition of the spleen was doubtful, at any rate, it was not certainly enlarged; there was a trace of albumen in the urine; there were no typhoid spots. One of the fingers of the left hand was swollen from base to tip; there was a red swollen area in the left ankle, and the left shoulder was painful swollen area in the left ankle, and the left shoulder was painful of typhoid fever with independent cirrhosis was justifiable; and I concluded that he had not croupous pneumonia; and, though I gave attention to the possibility of malignant endocarditis, I thought in the absence of a murmur typhoid was much more probable.

As the nursing arrangements were unsatisfactory he was sent to the local hospital. He died within forty-eight hours, and after death was found to have septic endocarditis of the aortic valves, which were extensively diseased; the mitral valve was healthy. It is, of course, possible that the condition of the finger was the primary lesion; but the finger, shoulder-joint their condition post mortem I have no information.

The next two cases are interesting as showing the deceptive appearances which malignant endocarditis may present.

John D., 61 years old, was admitted into Guy's Hospital on October 8th, 1906 .

He had been invalided from the army about two years previously for heart disease; he had since worked in Covent Garden as a porter, and for the last six months in a tannery. He had rheumatic fever in 1901, and again eighteen months later; last year he was in Guy's Hospital for the same disease affecting many joints, and mitral regurgitation was present

The present illness began on October $6 \mathrm{th}$, when he had been boxing with another man, and having dressed almost immediately, he returned home, and went to bed about 11 o'clock. He says he was not severely punished in the match, and he felt quite fit when he went to bed, but waking on Sunday morning (the 7th) he felt stiffness in the knees and right shoulder. The pain increased during that day and the following, and he felt so il that he came to the hospital.

When seen in bed he had the facial appearance of one in pain, but his eyes were closed, and he lay on his back with the legs extended, taking no notice of or interest in his survoundings. However, he admitted pain in his forehead, the back of his neck, and his head. The right shoulder and right upper arm were painful on movement; and the right shoulder was slightly swollen in front. The tongue was slightly furred, but moist and firm; the teeth clean and regular. rather rigid, the spleen not palpable, the liver extending for rather rigid, the spleen not palpable, the liver extending for
1 in. below the costal margin. The chest was tender all over; resonance was normal; some sibilant rhonchus was heard on the right side, and crackling râles at the left base below the angle of the scapula as far forward as the posterior axillary line. The cardiac impulse was in the fifth space, 3 in. from the median line; the superficial cardiac dullness was slightly extended to the left, almost to the nipple line, and there was a
loud systolic blowing murmur, heard at the apex, in the axilla, and behind.

The urine was free from albumen and sugar. Few changes took place in the next few days; he was very taciturn, made few complaints, and generally when asked denied that he had any pain. Later, it was obvious that his mind was not clear, and pain. Later, it was obvious that his mind was not clear, and small red spots were seen on the knees, and on the 15th he had an abundant eruption of petechiae and dark papules over the left thigh, knees, wrist, and back. Some were obviously petechial, persisting on pressure; others were certainly only
papules. The motions were soft, pasty, yellow-brown. The papules. The motions were soft, pasty, yellow-brown. The able to remove him to a fever hospital; he died there a few days afterwards, and he was found post mortem to have malignant endocarditis.

CASE vi.

M. A. E. F., aged 19, was admitted on February 16th, 1966. She had St. Vitus's dance at 13 years at age, and her heart had been affected since.

On February 9th, she complained of pain and shivered; she then had diarrhoea, with yellowish-green very offensive stools about four times daily. She had been treated with milk diet, but she had vomited sometimes. On the 15th she became

On her arrival in the ward the resemblance to meningitis was very close. Tache cérébale was slowly developed, but well
marked. The abdomen was flat, but not hollowed. "There were numerous flea-bites, and the hair was full of pediculi. She was drowsy, but responded to questions. The pupils were contracted, and there was a slight variable squint. The right arm was rather rigid. She was unable or unwilling to raise it, and then she tried to lift it with the left hand; afterwards she lifted it alone. On moving the arm she complained of some pain in ment of the spleen. The face was dusky and flushed; the ment of the spleen. The face was dusky and flushed; the breathing of diabetic coma. Many rhonchi were heard in the chest. No cardiac murmur was heard; but one must admit that a slight one may have been obscured by the rhonchi.

On the following iday, February 17th, she had convulsions of the left side of her body. She died at 2 p.m.

The post-mortem examination was made by Dr. French, and the notes are as follows: There were many minute petechiae in both white and grey matter of the brain; these were widely distributed, and not limited to any part. There was no cerebral softening, no meningitis, no large haemorrhage, and no other evidence of embolism. There were many scattered ecchymoses under the pleura posteriorly, but no pleurisy, old or recent. Red indurated lungs. No atheroma of pulmonary arterioles, no infarcts, no haemorrhages, and no oedema. Heart: Petechiae the pulmonary artery so that it was just twice the girth of the the pulmonary artery so that it was just twice the girth of the aorta. Both right ventricle and auricle dilated and hyperin circumference. Left auricle greatly dilated, not much in circumference. Left auricle greatly dilated, not much stenosed, but with soft edges; the orifice admitted two fingertips. The valve was thickened, the chordae thick and reduced in number, and there were irregular fungations on the valve at several spots ; these were spreading down some of the chordae,
but the fungations had not actually caused any ulceration of the valve.

The abdominal viscera were congested but presented no ecchymoses. Liver, natural in consistence, colour, and size. Pancreas, cyanosed. Spleen, soft, no infarcts, not enlarged, presenting some old capsulitis. Kidneys: Large organs, each nearly twice the size expected; many petechiae, both subcapsular and parenchymatous; the appearance suggested capsular and parenchymatous; the appearance suggested acute nephritis of recent date, together with ecchymoses. nephritis with congestion and cloudy swelling; no small roundnephritis with congestion and cloudy swelling; no small roundcelled exudation, in one place a thrombosed vessel. Dr. Louisson examined the blood bacteriologically, and stated that
the Bacillus coli only was present, and no rheumatic diplococci were detected.

\section{Case vir.}

G. T., aged 26, was transferred from a surgical ward on November 4th to the clinical ward. He had been admitted originally on October 20th, 1906, for a perineal fistula following ancess. A month previously he had been infected with was observed two weeks later; it was opened, but his temperature remained high, and he came to the hospital. On admission he was drowsy, and little history could be obtained. The temperature was high, there were two rigors on the first day, and there was a little discharge from the urethra. On day, and there was a little discharge from the urethra. On october 30 th he was found to have an aortic bruit, and signs
of liquid at the base of each chest. There was no history of rheumatic fever, and he had always been healthy.

Condition on November 4th: The patient is very anaemic, can lie in any position, and does not show much dyspnoea; unwilling to answer questions. Impulse of the heart in the fifth space, half an inch inside the nipple line. ' Precordial dullness reaches the upper border of the third rib above, the nipple line to the left and just beyond the border of the sternum. : There is no thrill. There is a to-and-fro murmur in the aortic area heard down the left border of the sternum as far as the impulse and upwards in both sides of the neck. Both sides of the chest move equally, but there is dullness at both bases, with impairment of breath sounds, vocal resonance, and tactile fremitus. There is no rub, nor râle, nor rhonchus. The pulse is aortic in character.

The following day the legs were oedematous. Two attempts to make a culture and vaccine, in the view that the infection was gonorrhoeal, were unsuccessful.

Later some clear brown fluid was drawn off from the left chest, but a cultivation was again negative. On November 11th a pericardial rub was heard, and the pleural fluid appeared to be increasing on the right side, and subsequently the dullness reached round to the front so as to be continuous with the heart's dullness. He died rather suddenly on November 16th: The post-mortem examination showed fungating endocarditis of the aortic valves, one of which was perforated, haemorrhages under the endocardium of the right auricle, haemorrhagic perithe left side, clear fluid in the right pleural cavity, and a recent infarct in the spleen.

A cultivation of some blood taken from the heart gave pneumococci.

This case is again difficult to classify. The gonorrhoeal infection was to all appearances the source of the trouble, 
and the endocarditis was secondary. It might be regarded as pyaemic or as cardiac; but the rigors were only few in number, and the thoracic symptoms were mainly due to pericardial and pleural effusions.

In conclusion, I will mention a case which $I$ saw with Dr. Marshall of Huddersfield as illustrating a condition of illness which led me to suspect a septic process, although no fever was at that time present.

\section{Case virI.}

A gentleman under 40 years of age lost flesh in March, found he could not go uphill with ease, and became sallow. A systolic apex bruit was discovered, and the urine contained albumen. Under treatment the albumen diminished. I saw him two months later on June 1st. He was pale and waxy in appearance, with a little flush on the cheeks. The heart's apex was in the nipple line, and there was a systolic murmur audible here, reaching into the axilla. The pulse was 120, rather full and hard. The liver was felt as low as the umbilicus level; the spleen was not felt, but there was pronounced dullness in the spleen was not felt, but there was pronounced dullness in the usual position; the urine now contained a good amount of perspiration at night, occurring twice or thrice weekly. The perspiration at night, occurring tor

optic discs and fundi were normal. Though there was no fever recorded, and though he had improved under treatment, I suspected malignant endocarditis, for the following reasons: His anaemia, wasting, loss of appetite, and sweating at night; a cardiac valvular lesion without the albumen in the urine, also out of proportion to the evidence of cardiac incompetence.

He went to the South Coast for a short time without material benefit; he returned home, and towards the end of July pyrexia developed.

I saw him again on August 11th at his home. He was very ill there was a double murmur at the base and at the apex, rîles abundant at the left base, and an enlarged liver. Injections of antistreptococcin had been given. He continued to have high temperatures at night, and he died about a fortnight later.

Here there was a case in which febrile temperature was only observed four or five weeks before death; though it is probable, if my view be accepted, that he was suffering from microbic infection in connexion with his cardiac valves for at least two months before that. Cases have been recorded in which even to the time of death pyrexia has been absent, and in such a case as that recorded above it can be only a matter of conjecture what determined the occurrence of pyrexia at one time rather than another. No doubt, in a great many cases, the onset of subjective sensations of illness coincides with the beginning of the pyrexia.

Other cases could be recited showing the great variety in the symptoms, course, and duration of this disease. But I think I have said enough to show that the familiar divisions do not cover the whole of the ground, and that many cases might be easily placed in two, or even in three, of the divisions.

What is important is to bear in mind that this disease is a form of pyrexial complaint which may be easily confounded with typhoid fever, tuberculosis, pneumonia, or other severe infection; that it has no one definite course or duration; that, as a rule, it is characterized by a valvular lesion, by pyrexia, by other signs of general infection, and by the evidences of vascular obstruction or embolism; that in diffeient cases pyrexia may be absent or murmurs may be absent, or there may be noevidence of vascular obstruction; that while some cases resemble typhoid fever, others pyaemia, in others pyrexia is grafted on marked cardiac symptoms, in others cerebral symptoms are prominent; there are cases which can come under neither of these groups, and others which may be included in two or three.

Tendering For PUBLIC ApPoIntments.-The following resolution was unanimously passed at a special meeting of the Council of the Society of Public Analysts and other Analytical Chemists on October 1st: "The Council of the Society of Public Analysts and other Analytical Chemists has had under consideration the practice which has been adopted recently by certain public bodies of inviting applicants for the office of public analyst to state the terms upon which they are prepared to accept such appointments. The council is of opinion that it is against the interests of the public and degrading to the profession of analytical chemistry for appointments to be 'offered 'on tender,' and calls upon the members of the society to resist the practice."

\section{BEES' STINGS AND RHEUMATISM.}

By E. W. AINLEY WALKER, D.M.Oxon., FELLOW AND TUTOR OF UNIVERSITY COLLEGE, AND LECTURER IN
PATHOLOGY IN THE UNIVERITY OF OXFORD.

IN consequence of certain observations previously recorded, ${ }^{1}$ which seemed to suggest a possible relationship between some of the phenomena of acute rheumatism and an abnormal production of formic acid in the body under the action of a streptococcal micro-organism, the attention of the writer was directed to the alleged popular belief that the poison of bees is protective against "rheumatism," and may also be successfully employed for its cure.

An inquiry was therefore set on foot with the object of ascertaining:

1. The extent to which this belief is prevalent.

2. The amount and kind of evidence available in its favour.

3. The amount of evidence which could be obtained to the contrary.

4. The clinical characters of the "rheumatism" thus stated to be cured.

A series of questions was published in the Lancet and the British Medical Journal on June 22nd, 1907, and subsequently in Nature and the British Bee Journal. They appear to have been widely copied into various newspapers in most English-speaking countries; and as the result the writer has engaged in a considerable correspondence with persons, both lay and medical, who have either recorded their own experience and information, or sent references to statements in the press bearing on the subject.

I was also fortunate enough to succeed in getting into correspondence with Dr. Terc, an Austrian physician of Marburg (Styria), whose observations are referred to in some of the press notices, and who has carried out the bee-sting treatment for many years past.

Apart from Dr. Terc's results, which appear to be worthy of special consideration, and must be referred to later, the material collected cannot be regarded as establishing anything beyond the fact that a widespread belief in the efficacy of the bee's sting for " rheumatism" exists. But it is highly suggestive, and should be sufficient to justify the removal of the subject from the realm of mere folklore into the sphere of practical investigation. But all that the writer has at present the opportunity of doing is to submit the statements which he has collected on the subject to the consideration of those who have facilities for clinical research.

It will be noted that the conditions referred to in the evidence which follows are for the most part what may be classed as " chronic rheumatism," but that the inquiry as to the actual characters of the affection concerned failed in nearly all cases to elicit any very satisfactory statement as regards its clinical features.

LETTERS FROM MEDICAL MEN.

1. Dr. W. Hammond (Cornwall) states that the belief is quite common in Cornwall ; knows no facts bearing on it.

2. Dr. W. R. Gore (Wales) had a patient who started keeping bees as a cure for rheumatism, and stated that the stings

cure him; knows that the belief is common in Shropshire. 3. Dr. H. P.

4. Dr. Boswell Dodds (Devon): The belief is common in Switzerland, where also the peasants in the Canton of Berne say that extract of ants is equally useful. His wife's godfather say that extract of ants is equally useful. His wife's godfather used to cure his rheumatism by making bees sting him. Also refers to a cast

bees' stings. prevalent in the States. Has seen the treatment applied in a case of acute rheumatism (temperature $103^{\circ} \mathrm{F}$., etc.), with the result that in twenty-four hours the temperature was normal and the joint free from pain and freely movable. The same patient (Colonel W. T.), informs him that he has had one subsequent attack, similarly cured.

6. Dr. R. McLay (Lincolnshire) mentions a case of rheumatism of the shoulder (stiff and painful) of two or three months' standing, cured by an accidental sting of the hand.

7. Dr. Stawell (Australia) knows of the belief, and has met a man who claimed to have been cured by bee stings.

man who claimed to have been cured by bee stings. 8. Dr. Valentine Rees (Wales) saw a case of lumbago treated
by bee stings applied to the back; the patient was then able to by bee stings applied to the back; the patient was then able to walk about with ease and comfort, which he had not been able
to do for weeks previously, but the condition returned in a few to do for weeks previously, but the condition returned in a few
days. The clergyman who applied the treatment stated that days. The clergyman who applied the treatment stated that since he began to keep bees. 